\title{
On the Osculatory Rational Interpolation Problem
}

\author{
By Luc Wuytack
}

\begin{abstract}
The problem of the existence and construction of a table of osculating rational functi. ns $r_{1, m}$ for $1, m \geqslant 0$ is considered. First, a survey is given of some results from the th sory of osculatory rational interpolation of order $s_{i}-1$ at points $x_{i}$ for $i \geqslant 0$. Using these results, we prove the existence of continued fractions of the form

$$
\begin{aligned}
c_{0}+c_{1} \cdot\left(x-y_{0}\right) & +\ldots+c_{k} \cdot\left(x-y_{0}\right) \ldots\left(x-y_{k-1}\right) \\
& +\frac{\left.c_{k+1} \cdot\left(x-y_{0}\right) \ldots\left(x-y_{k}\right)\right]+}{1}+\frac{c_{k+2} \cdot\left(x-y_{k+1}\right)}{1} \\
& +\frac{\left.c_{k+3} \cdot\left(x-y_{k+2}\right)\right]}{1}+\ldots,
\end{aligned}
$$
\end{abstract}

with the $y_{k}$ suitably selected from among the $x_{i}$, whose convergents form the elements $r_{k, 0}, r_{k+1,0}, r_{k+1,1}, r_{k+2,1}, \ldots$ of the table. The properties of these continued fractions make it possible to derive an algorithm for constructing their coefficients $c_{i}$ for $i \geq 0$. This algorithm is a generalization of the $q d$-algorithm.

1. Introduction. Let $X$ be a set of distinct points $x_{i}$ and $s_{i}$ be a positive integer for $i \geqslant 0$. Let $f$ be a real function, whose derivatives $f_{i}^{(k)}$ for $k=0,1, \ldots, s_{i}-1$ are given in each of the points $x_{i}$. The class of polynomials of degree at most $n$ will be denoted by $P_{n}$ for every $n \geqslant 0$.

Let $l$ and $m$ be nonnegative integers such that $l+m=\sum_{i=0}^{j} s_{i}+t-1$ and $1 \leqslant$ $t \leqslant s_{j+1}$. The class of (ordinary) rational functions $r=p / q$, with $p \in P_{l}, q \in P_{m}$ and $p / q$ irreducible, will be denoted by $R(l, m)$. The problem of osculatory rational interpolation of order $(l, m)$ consists in finding an element $r$ in $R(l, m)$ whose derivatives satisfy the following relations:

$$
\begin{aligned}
r^{(k)}\left(x_{i}\right) & =f_{i}^{(k)} \quad \text { for } k=0,1, \ldots, s_{i}-1 \text { and } i=0,1, \ldots, j, \\
r^{(k)}\left(x_{j+1}\right) & =f_{j+1}^{(k)} \quad \text { for } k=0,1, \ldots, t-1 .
\end{aligned}
$$

In order to answer the question about the existence and uniqueness of a solution for this problem, we also consider the problem of finding elements $p \in P_{l}$ and $q \in P_{m}$ satisfying

$$
p^{(k)}\left(x_{i}\right)=(f \cdot q)^{(k)}\left(x_{i}\right) \quad \text { for } k=0,1, \ldots, s_{i}-1 \text { and } i=0,1, \ldots, j,
$$

$$
p^{(k)}\left(x_{j+1}\right)=(f \cdot q)^{(k)}\left(x_{j+1}\right) \text { for } k=0,1, \ldots, t-1 .
$$

Let $p(x)=\Sigma_{i=0}^{l} a_{i} \cdot x^{i}$ and $q(x)=\Sigma_{i=0}^{m} b_{i} \cdot x^{i}$; then (2) can be considered as a system of $l+m+1$ linear homogeneous equations in the $l+m+2$ unknowns $a_{0}, a_{1}, \ldots$, $a_{l}, b_{0}, \ldots, b_{m}$. Consequently, this system has always at least one nontrivial solution. Let $p_{1}, q_{1}$ and $p_{2}, q_{2}$ be two different solutions of (2) and define $r_{1}$ and $r_{2}$ as follows: 
$r_{1}=p_{1} / q_{1}$ and $r_{2}=p_{2} / q_{2}$. It is not hard to prove that $r_{1}$ and $r_{2}$ are equivalent, or $p_{1} \cdot q_{2}=p_{2} \cdot q_{1}$.

Let $p, q$ be a solution of (2); then $r=p / q$ may not be an element of $R(l, m)$ since $p / q$ might be reducible. Let $p_{0} / q_{0}$ be the irreducible form of $p / q$. If $q_{0}=$ $\sum_{i=0}^{m} d_{i} \cdot x^{i}$ with $d_{j} \neq 0$ and $d_{i}=0$ for $i=0,1, \ldots, j-1$, then we assume that $p_{0} / q_{0}$ is normalized in such a way that $d_{j}=1$. The rational function $r_{l, m}$ in $R(l, m)$ defined by $r_{l, m}(x)=p_{0}(x) / q_{0}(x)$ for every $x$ with $q_{0}(x) \neq 0$, is called the $(l, m)$-osculant of $f$. The exact degree of $p_{0}$ and $q_{0}$ will be denoted by $l^{\prime}$ and $m^{\prime}$ respectively.

In the next section, some results on the existence and uniqueness of a solution to the system (1) are given. A necessary and sufficient condition for the existence of such a solution is proved, and it is shown that there is at most one rational function in $R(l, m)$ satisfying (1).

In Section 3, sequences of osculating rational functions of different degree are considered. The existence of continued fractions, whose convergents form the elements of these sequences, is shown.

Algorithms for computing sequences of osculating rational functions are considered in Section 4. A new algorithm is derived, based on the properties of osculating continued fractions. It is shown in Section 5 that this algorithm is a generalization of the $q d$-algorithm for Padé approximation.

The basic ideas used in this paper are similar to those used in [6] and [7] for the case of ordinary rational interpolation.

\section{Existence and Uniqueness of a Solution in Osculatory Rational Interpolation.}

Since the rational functions associated with two solutions of (2) are equivalent, they have the same irreducible form. Therefore the definition of $(l, m)$-osculant implies the following result.

LEMMA 1. Let $l$ and $m$ be nonnegative integers; then there exists a unique $(l, m)$ osculant of $f$.

Consider the $(l, m)$-osculant $r_{l, m}=p_{0} / q_{0}$ of $f$. Using a simple example we now show that $p_{0}$ and $q_{0}$ do not always satisfy Eqs. (2).

Example 1. Let $x_{0}=0, x_{1}=1, s_{0}=2, s_{1}=1$ and $f_{0}^{(0)}=f_{0}^{(1)}=f_{1}^{(0)}=1$. Consider the problem of finding elements $p, q \in P_{1}$ satisfying (2) or

$$
p\left(x_{0}\right)=f_{0}^{(0)} \cdot q\left(x_{0}\right), \quad p^{\prime}\left(x_{0}\right)=f_{0}^{(1)} \cdot q\left(x_{0}\right)+f_{0}^{(0)} \cdot q^{\prime}\left(x_{0}\right), \quad p\left(x_{1}\right)=f_{1}^{(0)} \cdot q\left(x_{1}\right) .
$$

The elements $p$ and $q$ defined by $p(x)=q(x)=x$ for all $x$ satisfy these equations. Consider $p_{0}=q_{0}=1$; then the $(1,1)$-osculant of $f$ is defined by $r_{1,1}=p_{0} / q_{0}$. It is, however, clear that $p_{0}$ and $q_{0}$ do not form a solution of the above equations.

Using the $(l, m)$-osculant $r_{l, m}=p_{0} / q_{0}$ of $f$ it is possible to construct a solution of (2) very easily by multiplying $p_{0}$ and $q_{0}$ with certain powers of $\left(x-x_{j}\right)$ for some $j \geqslant 0$. To do this more explicitly, we introduce the following notations. Let the points $y_{0}, y_{1}, y_{2}, \ldots$ be defined in the following way

$$
\begin{aligned}
& y_{i}=x_{0} \text { for } i=0,1, \ldots, s_{0}-1 \quad \text { and } \\
& y_{s+i}=x_{j} \quad \text { for } i=0,1, \ldots, s_{j}-1, \quad \text { with } s=\sum_{k=0}^{j-1} s_{k} \text { and } j \geqslant 1 .
\end{aligned}
$$


The next lemma can now be proved by applying a method similar to the one used in the proof of Lemma 2 in [7].

Lemma 2. If $r_{l, m}=p_{0} / q_{0}$ is the $(l, m)$-osculant of $f$, then there exist $N$ points $\left\{z_{1}, z_{2}, \ldots, z_{N}\right\}$ in $\left\{y_{0}, y_{1}, \ldots, y_{l+m}\right\}$, with $0 \leqslant N \leqslant \min \left\{l-l^{\prime}, m-m^{\prime}\right\}$, such that $p=\left(x-z_{1}\right) \ldots\left(x-z_{N}\right) \cdot p_{0}$ and $q=\left(x-z_{1}\right) \ldots\left(x-z_{N}\right) \cdot q_{0}$ satisfy Eqs. (2).

In order to apply Lemma 2 to the situation given in Example 1, we take $N=1$ and $z_{1}=x_{0}$. Since $r_{1,1}=p_{0} / q_{0}$ with $p_{0}=q_{0}=1$, we have $l^{\prime}=m^{\prime}=0$ and $\min \left(l-l^{\prime}, m-m^{\prime}\right)=1$. The elements $p_{0}$ and $q_{0}$ do not satisfy Eqs. (2), but it is clear that $p=\left(x-x_{0}\right) \cdot p_{0}$ and $q=\left(x-x_{0}\right) \cdot q_{0}$ form a solution of (2).

The following important relationship between the systems (1) and (2) was proved by H. E. Salzer in [3, p. 487].

LEMMA 3. If $q\left(x_{i}\right) \neq 0$, then the system

$$
(p / q)^{(k)}\left(x_{i}\right)=f^{(k)}\left(x_{i}\right) \text { for } k=0,1, \ldots, s_{i}-1,
$$

is equivalent to the system

$$
(p)^{(k)}\left(x_{i}\right)=(f \cdot q)^{(k)}\left(x_{i}\right) \text { for } k=0,1, \ldots, s_{i}-1 .
$$

In the remaining part of this paper, we say that an element $r=p / q$ from $R(l, m)$ is satisfying (2) if $p$ and $q$ satisfy (2). It is now possible to give a necessary and sufficient condition for the existence of a solution for (1).

THEOREM 1. There exists a rational function $r$ in $R(l, m)$ satisfying $(1)$ if and only if the $(l, m)$-osculant of $f$ satisfies (2).

Proof. Let $r=p / q$ be a solution of (1) then $q\left(x_{i}\right) \neq 0$ for $i=0,1, \ldots, j+1$. Using Lemma 3 we find that Eqs. (2) hold. If $r$ is an element of $R(l, m)$, then $p / q$ is irreducible. Since $r_{l, m}$ is unique (Lemma 1 ), we must have $r_{l, m}=p / q$, consequently the $(l, m)$-osculant satisfies (2). The converse can be proved as follows. Suppose $r_{l, m}=$ $p_{0} / q_{0}$; then the irreducibility of $p_{0} / q_{0}$ implies $q_{0}\left(x_{i}\right) \neq 0$ for $i=0,1, \ldots, j+1$. If $r_{l, m}$ satisfies (2), then Lemma 3 implies that $p_{0} / q_{0}$ is a solution of (1), or there exists an element in $R(l, m)$ satisfying (1).

From the proof of Theorem 1 we see that every element $r$ in $R(l, m)$ satisfying (1) must be equal to $r_{l, m}$. Consequently, Lemma 1 and Theorem 1 imply the following result.

THEOREM 2. If the osculatory rational interpolation problem (1) has a solution in $R(l, m)$, then this solution is unique and equal to $r_{l, m}$.

3. Osculatory Continued Fractions. The $(l, m)$-osculants of $f$, for different values of $l$ and $m$, can be arranged in a scheme like Table 1 .

$$
\begin{array}{ccccc}
r_{0,0} & r_{0,1} & r_{0,2} & r_{0,3} & \cdots \\
r_{1,0} & r_{1,1} & r_{1,2} & r_{1,3} & \ldots \\
r_{2,0} & r_{2,1} & r_{2,2} & r_{2,3} & \ldots
\end{array}
$$

TABLE 1. Table of $(l, m)$-osculant: of $f$ 
We assume that every element in Table 1 is a solution of the corresponding osculatory rational interpolation problem (1). As a corollary of Lemma 2, this condition holds, for example, in the case where the elements $r_{l, m}$ of Table 1 are regular, or $\min \left\{l-l^{\prime}, m^{\prime}-m^{\prime}\right\}=0$.

In the remaining part of this paper, continued fractions of the following form will be considered

$$
\begin{aligned}
c_{0}+c_{1} \cdot\left(x-y_{0}\right)+\ldots+c_{k} \cdot\left(x-y_{0}\right) \ldots\left(x-y_{k-1}\right) \\
+\frac{c_{k+1} \cdot\left(x-y_{0}\right) \ldots\left(x-y_{k}\right)}{1}+\frac{c_{k+2} \cdot\left(x-y_{k+1}\right)}{1} \\
++\frac{\left.c_{k+3} \cdot\left(x-y_{k+2}\right)\right]+\ldots,}{1}+\ldots
\end{aligned}
$$

where the points $y_{i}$ for $i \geqslant 0$ are defined by (3). Let $T_{k}$ be a set of elements lying on a staircase in Table 1 , or $T_{k}=\left\{r_{k, 0}, r_{k+1,0}, r_{k+1,1}, r_{k+2,1}, \ldots\right\}$ for $k \geqslant 0$. We will show that the coefficients $c_{i}$ for $i \geqslant 0$ in (4) can be defined in such a way that the convergents of (4) are the elements of $T_{k}$. First we prove a relationship between certain elements in Table 1.

LemMA 4. Let $v(x)=\left(x-y_{0}\right) \cdot\left(x-y_{1}\right) \ldots\left(x-y_{l+m}\right), r_{l, m}=p_{1} / q_{1}$ and $r_{l+\mu, m+\nu}=p_{2} / q_{2}$ with $\mu, \nu \geqslant 0$. There exists a polynomial $w$ of degree at most $\max \{\mu-1, \nu-1\}$ such that $p_{1} \cdot q_{2}-p_{2} \cdot q_{1}=v \cdot w$.

Proof. Let $j$ and $t$ be such that $l+m=\sum_{i=0}^{j} s_{i}+t-1$ and $1 \leqslant t \leqslant s_{j+1}$. Since we assumed that $r_{l, m}$ and $r_{l+\mu, m+\nu}$ are solutions of (1), we get that $p_{1} / q_{1}$ and $p_{2} / q_{2}$ are solutions of (2) by applying Lemma 3. The relation $p_{1} \cdot q_{2}-p_{2} \cdot q_{1}=$ $\left(p_{1}-f \cdot q_{1}\right) \cdot q_{2}-\left(p_{2}-f \cdot q_{2}\right) \cdot q_{1}$ then implies

$$
\begin{aligned}
\left(p_{1} \cdot q_{2}-p_{2} \cdot q_{1}\right)^{(k)}\left(x_{i}\right)=0 & \text { for } k=0,1, \ldots, s_{i}-1 \text { and } i=0,1, \ldots, j, \\
\left(p_{1} \cdot q_{2}-p_{2} \cdot q_{1}\right)^{(k)}\left(x_{j+1}\right)=0 & \text { for } k=0,1, \ldots, t-1 .
\end{aligned}
$$

Consequently, there exists a polynomial $w$ such that $p_{1} \cdot q_{2}-p_{2} \cdot q_{1}=v \cdot w$. Since the degree of $p_{1} \cdot q_{2}-p_{2} \cdot q_{1}$ is at most $l+m+\max \{\mu, \nu\}$, we get that the degree of $w$ can be at most $\max \{\mu-1, \nu-1\}$.

THEOREM 3. If the elements of $T_{k}$ are different from each other, then there exists a continued fraction of the form (4), with $c_{k+i} \neq 0$ for $i \geqslant 1$ whose convergents are the elements of $T_{k}$.

Proof. Let $r_{k+i, j}=p_{i+j} / q_{i+j}$ for $i=j, j+1$ and $j=0,1,2, \ldots$ We construct a continued fraction of the form

$$
\beta_{0}+\frac{\left.\alpha_{1}\right]}{\left[\beta_{1}\right.}+\frac{\alpha_{2} \mid}{\left[\beta_{2}\right.}+\frac{\alpha_{3}}{\left[\beta_{3}\right.} \ldots
$$

whose $n$th convergent is equal to $p_{n} / q_{n}$ for $n \geqslant 0$. This means that $\beta_{0}$ and $\alpha_{i}, \beta_{i}$ for $i>0$ have to satisfy the relations

$$
\begin{aligned}
& \beta_{0}=p_{0}, \quad \beta_{0} \cdot \beta_{1}+\alpha_{1}=p_{1}, \quad \beta_{1}=q_{1}, \\
& \left.\begin{array}{l}
\beta_{n} \cdot p_{n-1}+\alpha_{n} \cdot p_{n-2}=p_{n} \\
\beta_{n} \cdot q_{n-1}+\alpha_{n} \cdot q_{n-2}=q_{n}
\end{array}\right\} \text { for } n \geqslant 2 .
\end{aligned}
$$


Consequently $\beta_{0}=p_{0}, \beta_{1}=1$ and $\alpha_{1}=p_{1}-p_{0}$. These equations and the definitions of $p_{0}$ and $p_{1}$ imply the existence of coefficients $c_{0}, c_{1}, \ldots, c_{k+1}$ such that

$$
\begin{aligned}
& \beta_{0}=c_{0}+c_{1} \cdot\left(x-y_{0}\right)+\ldots+c_{k} \cdot\left(x-y_{0}\right) \ldots\left(x-y_{k-1}\right) \text { and } \\
& \alpha_{1}=c_{k+1} \cdot\left(x-y_{0}\right) \ldots\left(x-y_{k}\right) .
\end{aligned}
$$

Since $r_{k, 0} \neq r_{k+1,0}$ we get $p_{1} \neq p_{0}$; consequently, $c_{k+1} \neq 0$. Using (6) and Lemma 4, we get the existence of nonzero constants $a_{n}, b_{n}$ such that

$$
\alpha_{n}=a_{n} \cdot\left(x-y_{k+n-1}\right) \text { and } \beta_{n}=b_{n} \text { for } n \geqslant 2
$$

By using an equivalence transformation, the continued fraction (5) can be reduced to a continued fraction of the form (4) with $c_{k+i} \neq 0$ for $i \geqslant 1$. This concludes the proof.

The elements of $T_{k}$ for $k \geqslant 0$ form the lower triangular part of Table 1. A theorem, similar to Theorem 3 , can also be given about the existence of continued fractions whose convergents form a staircase $\left\{r_{0, k}, r_{0, k+1}, r_{1, k+1}, r_{1, k+2}, r_{2, k+2}, \ldots\right\}$ in the upper triangular part of Table 1.

4. The Computation of Osculating Rational Functions. In order to compute an element $r_{l, m}$ in Table 1 , several methods are already available. In most cases, a sequence $T_{k}$ is constructed until $r_{l, m}$ is reached, where the value of $k$ must be chosen in such a way that $r_{l, m}$ is one of the elements of $T_{k}$.

Methods to compute $T_{0}$ are given by Thiele [2] and Salzer [3]. In Thiele's method, reciprocal differences with confluent arguments are used, while Salzer's method gives a more direct approach avoiding confluent reciprocal differences. To compute $T_{k}$ for $k \geqslant 0$, methods of Thacher [5] and Kahng [1] are available. Thacher's method is based on the use of recurrence relations between the numerators and denominators of consecutive elements in $T_{k}$. In Kahng's method, a certain type of divided differences is used.

We will now describe an algorithm to compute the lower half of Table 1, by using certain relations between the sequences $T_{k}$ and $T_{k+1}$. A similar method can be given to construct the upper half of this table. Consider the following continued fraction $g_{k}$ for $k \geqslant 0$ :

$$
\begin{aligned}
& g_{k}(x)=c_{0}+c_{1} \cdot\left(x-y_{0}\right)+\ldots+c_{k} \cdot\left(x-y_{0}\right) \ldots\left(x-y_{k-1}\right) \\
& +\frac{c_{k+1} \cdot\left(x-y_{0}\right) \ldots\left(x-y_{k}\right) \mid}{1}-\frac{q_{1}^{k+1} \cdot\left(x-y_{k+1}\right)}{1} \\
& -\frac{e_{1}^{k+1} \cdot\left(x-y_{k+2}\right) \mid}{\mid}-\frac{q_{2}^{k+1} \cdot\left(x-y_{k+3}\right) \mid}{\Gamma}-\ldots
\end{aligned}
$$

If the elements of $T_{k}$ are different from each other, then it follows from Theorem 3 that the coefficients in $g_{k}$ can be defined in such a way that the $n$th convergent $g_{k, n}$ of $g_{k}$ is equal to the $n$th element of $T_{k}$. Using contraction [4, p. 389], it is possible to obtain a continued fraction $h_{k}$ whose convergents $h_{k, n}$ satisfy the relation $h_{k, n}=$ $g_{k, 2 n+1}$ for $n \geqslant 0$. If we also consider $g_{k+1}$, then $h_{k+1}$ can be defined such that $h_{k+1, n}=g_{k+1,2 n}$ for $n \geqslant 0$. It is clear that the convergents of $h_{k}$ and $h_{k+1}$ represent the same rational functions, namely, $r_{k+1,0}, r_{k+2,1}, r_{k+3,2}, \ldots$ for $k \geqslant 0$. By identifying 
the corresponding coefficients in $h_{k}$ and $h_{k+1}$, after normalizing, we get the following relations for $k \geqslant 1$ :

$$
q_{1}^{k}=\frac{1}{d_{1}^{k}} \cdot \frac{c_{k+1}}{c_{k}}, \quad e_{1}^{k}=\frac{1}{d_{1}^{k}} \cdot q_{1}^{k+1}-q_{1}^{k}
$$

and for $i \geqslant 2$ :

$$
q_{i}^{k}=\frac{d_{i-2}^{k}}{d_{i}^{k}} \cdot\left[q_{i-1}^{k+1} \cdot \frac{e_{i-1}^{k+1}}{e_{i-1}^{k}}\right], \quad e_{i}^{k}=\frac{d_{i-1}^{k}}{d_{i}^{k}} \cdot\left[e_{i-1}^{k+1}+q_{i}^{k+1}\right]-q_{i}^{k} .
$$

The normalizing constants $d_{i}^{k}$ satisfy the following relations for $k \geqslant 1$ :

$$
\begin{gathered}
d_{0}^{k}=1, \quad d_{1}^{k}=1+\frac{c_{k+1}}{c_{k}} \cdot\left[y_{k+1}-y_{k}\right], \\
d_{i}^{k}=d_{i-1}^{k} \cdot\left[1+z_{k, i} \cdot e_{i-1}^{k+1 \eta}\right]-d_{i-2}^{k} \cdot z_{k, i} \cdot\left[q_{i-1}^{k+1} \cdot \frac{e_{i-1}^{k+1}}{e_{i-1}^{k}}\right] \\
\text { with } z_{k, i}=y_{k+2 i-2}-y_{k+2 i-1} \text { for } i \geqslant 2 .
\end{gathered}
$$

The elements $r_{k, 0}$ for $k \geqslant 0$ in the first column of Table 1 are osculating polynomials. This means that $c_{0}, c_{1}, c_{2}, \ldots$ can be computed by using divided differences with confluent arguments $[4$, p. 246]. In order to use the algorithm, a scheme of the form given in Table 2 can be constructed.

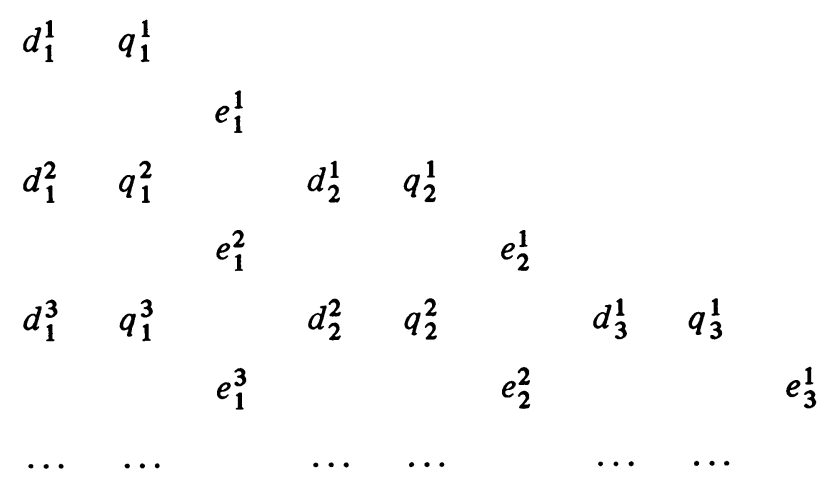

TABLE 2. Table of coefficients used in the algorithm

It is convenient to define the elements $e_{0}^{k}=0$ for $k \geqslant 1$, since in this case the formula (9) for $e_{i}^{k}$ can also be applied for $i=1$. The first and second columns in Table 2 can be computed by means of (10) and (8). In order to compute the other columns, the formulas (9) and (11) can be used. The above algorithm is now illustrated in a simple example.

Example 2. Let $x_{0}=0, x_{1}=1, s_{0}=s_{1}=2$ and $f_{0}^{(0)}=1, f_{0}^{(1)}=f_{1}^{(0)}=2$, $f_{1}^{(1)}=3$. Using divided differences with confluent arguments, we get $c_{0}=1, c_{1}=2$, $c_{2}=-1$ and $c_{3}=3$. The corresponding elements in Table 2 are $d_{1}^{1}=1 / 2, q_{1}^{1}=-1$, $d_{1}^{2}=1, q_{1}^{2}=-3, e_{1}^{1}=-5$. Using these coefficients, we can form the osculating continued fractions $(7)$ whose convergents are the $(l, m)$-osculants of $f$. We get 


$$
\begin{array}{ll}
r_{0,0}=1, & r_{1,1}=\frac{1+3 \cdot x}{1+x}, \\
r_{1,0}=1+2 \cdot x, & \\
r_{2,0}=1+2 \cdot x-x^{2}, & \\
r_{3,0}=1+2 \cdot x-4 x^{2}+3 \cdot x^{3}, & r_{2,1}=\frac{\left(5 \cdot x^{2}-x-2\right) / 3}{x-2 / 3} .
\end{array}
$$

5. Special Cases. In the case of ordinary rational interpolation $s_{i}=1$ for $i=0,1$, $2, \ldots$ The corresponding theory and algorithm for this situation is given in [6] and [7].

In the case of Padé approximation, $y_{i}=x_{0}$ for $i \geqslant 0$, The relations (10) and (11) imply $d_{i}^{k}=1$ for all $i \geqslant 0$ and $k \geqslant 1$. The algorithm described by (8) and (9) is then identical with the $q d$-algorithm [4, p. 402].

Acknowledgment. The author would like to thank the referee for his helpful remarks in improving the readability of this paper.

\section{Department of Mathematics University of Antwerp \\ Universiteitsplein 1 \\ B-2610 Wilrijk, Belgium}

1. S. W. KAHNG, “Osculatory interpolation," Math. Comp., v. 23, 1969, pp. 621-630. MR 40 \#995.

2. L. M. MILNE-THOMSON, The Calculus of Finite Differences, Macmillan, London, 1933.

3. H. E. SALZER, "Note on osculatory rational interpolation," Math. Comp., v. 16, 1962, pp. 486-491. MR 26 \#7133.

4. R. SAUER et al., Mathematische Hilfsmittel des Ingenieurs. Teil III, Die Grundlehren der math. Wissenschaften, Band 141, Springer-Verlag, Berlin and New York, 1968. MR 37 \#7115.

5. H. C. THACHER, JR., "A recursive procedure for osculatory interpolation by rational functions." (Unpublished manuscript.)

6. L. WUYTACK, "An algorithm for rational interpolation similar to the $q d$-algorithm," Numer. Math., v. 20, 1972/73, pp. 418-424. MR 48 \#3222.

7. L. WUYTACK, "On some aspects of the rational interpolation problem," SIAM J. Numer. Anal., v. 11, 1974, pp. 52-60. 